\title{
A Groves Mechanism Approach to Decentralized Design of Supply Chains
}

\author{
D. Garg and Y. Narahari \\ Indian Institute of Science, Bangalore - 560 012, India. \\ dgarg, hari@csa.iisc.ernet.in \\ Earnest Foster, Devadatta Kulkarni, and Jeffrey D. Tew \\ Manufacturing Systems Research Laboratory \\ General Motors Research and Development, Warren, MI, USA \\ earnest.foster, datta.kulkarni, jeffrey.tew@gm.com
}

\begin{abstract}
In this paper, a generic optimization problem arising in supply chain design is modeled in a game theoretic framework and solved as a decentralized problem using a mechanism design approach. We show that the entities in a supply chain network can be naturally modeled as selfish, rational, and intelligent agents interested in maximizing certain payoffs. This enables us to define a supply chain design game and we show that the well known Groves mechanisms can be used to solve the underlying design optimization problem. We illustrate our approach with a representative three stage distribution process of a typical automotive supply chain.
\end{abstract}

\section{Introduction}

Traditionally, resource allocation problems have been approached in a centralized way. However, more recently, decentralized approaches, in particular, game theoretic approaches, have been suggested, for example, see the survey paper by Cachon and Netessine [1], the paper by Fan, Stallaert, and Whinston [3], and the papers by Kutanoglu and $\mathrm{Wu}[7,9]$. Our interest in this paper is on using a game theoretic and mechanism design oriented approach for solving supply chain design and optimization problems.

A supply chain network could be considered as a conglomeration of nearly autonomous entities which have their own objectives and utilities to maximize, which may not necessarily result in a social optimum for the overall network. Individual entities of a supply chain can be realistically modeled as rational, selfish, and intelligent agents trying to outwit one another so as to maximize individual goals and not reporting their true costs or values to any central design authority (or supply chain manager). An appropri- ate model for such a system is a non-cooperative game with incomplete information. Motivated by this, we argue in favor of a decentralized design paradigm for supply chains and propose a mechanism design approach for design of supply chains. Several authors have explored this line of thinking in recent times. For example, Fan, Stallaert, and Whinston propose a decentralized way of designing a supply chain organization [3] based on a multicommodity network flow formulation. The supply chain planning problem is solved by these authors using a combinatorial auction framework. In a series of recent papers, Kutanoglu and Wu [7, 9] have used Vickrey-Clarke-Groves (VCG) mechanisms [2] for solving distributed resource planning problems arising in semiconductor capacity allocation, electronics component manufacturing, etc.

\subsection{Contributions and Outline of the Paper}

The following are the specific contributions of the paper.

- We first present a generic optimization problem arising in supply chain design and show the traditional centralized way of solving this problem (Section 2).

- We define the supply chain design game as a Bayesian game with incomplete information, leading to a decentralized approach for supply chain design (Section 3).

- We propose a mechanism design approach based on Groves mechanisms for solving the supply chain design game. Based on this, we present an iterative algorithm for decentralized design (Section 4).

- We show the efficacy of the proposed approach and algorithm using a stylized case study of a three stage distribution process of an automotive supply chain (Section 5). 


\section{A Generic Supply Chain Design Optimiza- tion Problem}

The formulation here is on the same lines of [4]. Consider a make-to-order, linear supply chain with $n$ stages/partners/players. The processing time or lead time for an end customer order at any stage $i$ is a continuous random variable $T_{i}$. Let us assume that $T_{i} ; i=1, \ldots, n$ are independent random variables and there is no time elapsed between the end of process $i$ and commencement of process $i+1(i=1, \ldots, n-1)$. We assume that $T_{i}$ is normally distributed with mean $\mu_{i}$ and standard deviation $\sigma_{i}$. Under these assumptions, the end-to-end lead time $Y$ of unit order for the supply chain is given by $Y=\sum_{i=1}^{n} T_{i}$. $Y$ is normally distributed with mean $\mu=\sum_{i=1}^{n} \mu_{i}$ and variance $\sigma^{2}=\sum_{i=1}^{n} \sigma_{i}^{2}$ since it is the sum of $n$ independent Gaussian random variables. Let us assume that for any stage $i$, the parameters, $\mu_{i}$ and $\sigma_{i}$ can take values from known sets but otherwise are fixed. The cost of processing a single order at stage $i$ is given by the function $v_{i}\left(\mu_{i}, \sigma_{i}\right)$. Note that the function $v_{i}\left(\mu_{i}, \sigma_{i}\right)$ captures the cost versus delivery lead time tradeoff at stage $i$. Let us assume that a Central Design Authority (CDA) who is managing the overall supply chain needs to determine optimal values for the parameters $\mu_{i}$ and $\sigma_{i}$ of each stage $i$ so that a superior level of delivery performance is achieved at minimum cost. In what follows, we formally define what we mean by superior level of delivery performance. A more rigorous treatment can be found in [4].

We assume that the CDA's target is to deliver the orders to the respective customers within $\tau \pm T$ days of receiving the order. We call $\tau$ the target delivery date and $T$ the tolerance. We also define $L=\tau-T$ to be the lower limit of the delivery window and $U=\tau+T$ to be the upper limit of the delivery window. The process capability indices $C_{p}, C_{p k}$ and $C_{p m}$, which are popular in the areas of design tolerancing and statistical process control, can be used to measure the performance of the end-to-end delivery process $Y$. See $[6,8,4]$ for a comprehensive treatment of process capability indices. The three indices $C_{p}, C_{p k}$, and $C_{p m}$ for the end-toend delivery process $Y$ are defined in following way:

$$
\begin{aligned}
C_{p} & =\frac{U-L}{6 \sigma}=\frac{T}{3 \sigma} \\
C_{p k} & =\frac{\min (U-\mu, \mu-L)}{3 \sigma}=\left(\frac{d}{3 \sigma}\right) \\
C_{p m} & =\frac{U-L}{6 \xi}=\frac{T}{3 \sqrt{\sigma^{2}+b^{2}}}
\end{aligned}
$$

The yield of the end-to-end delivery process $Y$ plays a critical role in defining superior level of delivery performance. The yield is simply the probability of delivering an order within a specified interval $\tau \pm T$ and can be expressed in terms of its capability indices $C_{p}, C_{p k}$ and $C_{p m}$ in the following way [4].

$$
\text { Yield }=\Phi\left(3 C_{p k}\right)+\Phi\left(6 C_{p}-3 C_{p k}\right)-1
$$

where $\Phi($.$) is the cumulative distribution function of the$ standard normal distribution. It can be verified that a unique $\left(C_{p}, C_{p k}\right)$ pair results in a unique yield, therefore, the 3 tuple $\left(C_{p}, C_{p k}, C_{p m}\right)$ can be substituted by the pair (Yield, $C_{p m}$ ) for the purpose of measuring the delivery performance. Being an indicator for precision and accuracy of the deliveries, we prefer to call the yield of the process as Delivery Probability $(D P)$ and $C_{p m}$ as Delivery Sharpness $(D S)$.

\subsection{Supply Chain Design Optimization Problem (SCOP)}

The following parameters are known in a typical SCOP problem.

1. The delivery window $(\tau, T)$ as fixed by the CDA.

2. Mean $\mu_{i}$ of random variable $T_{i}, i=1,2, \ldots, n$.

3. DP and DS (or $C_{p m}$ ) for end-to-end lead time $(Y)$ as fixed by the CDA.

4. Cost functions $\mathcal{K}_{i}=v_{i}\left(\sigma_{i}\right)$ submitted by each stage $i$ to the CDA. The function $\mathcal{K}_{i}$ captures the cost incurred at stage $i$ for attaining a standard deviation of $\sigma_{i}$ in the processing time of unit order at stage $i$. For the sake of conceptual and computational simplicity, we choose a second order polynomial of the form:

$$
\mathcal{K}_{i}=A_{i 0}+A_{i 1} \sigma_{i}+A_{i 2} \sigma_{i}^{2}
$$

The decision variables of the SCOP problem are the standard deviations $\sigma_{i}^{*}$ of each individual stage $i(i=1, \ldots, n)$. The objective of the SCOP is to minimize the end-to-end delivery cost and the problem formulation becomes:

minimize

$$
\mathcal{K}=\sum_{i=1}^{n} \mathcal{K}_{i}=\sum_{i=1}^{n}\left(A_{i 0}+A_{i 1} \sigma_{i}+A_{i 2} \sigma_{i}^{2}\right)
$$

subject to

$$
\begin{aligned}
\text { DS for end-to-end lead time } & \geq C_{p m}^{*} \\
\text { DP for end-to-end lead time } & \geq D P^{*} \\
\sigma_{i} & >0 \forall i
\end{aligned}
$$

We focus on this optimization problem in the rest of this paper. 


\subsection{Centralized Design Paradigm}

In the centralized way of solving the SCOP problem, first the CDA invites each stage to submit its cost function. After receiving the true cost function from each stage, the CDA solves an optimization problem that will minimize the total expediting cost while ensuring a required level of delivery performance. The solution of this optimization problem yields the optimal values for the design parameters $\left(\sigma_{i}^{*}\right)$ which are communicated back to the respective stage by the CDA. This scheme is shown in Figure 1.

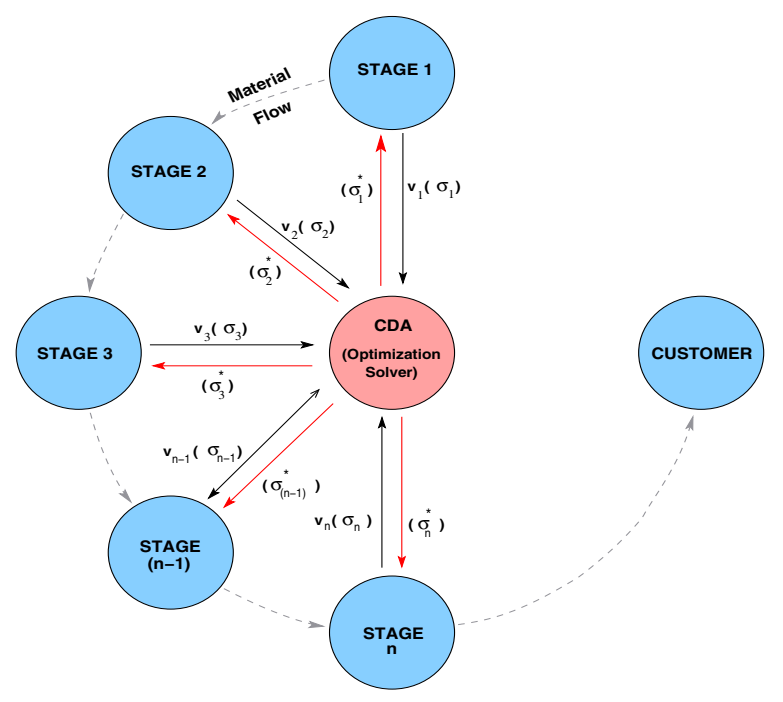

Figure 1. The centralized design paradigm

\section{Supply Chain Design Game}

A critical assumption in the centralized design paradigm is that each stage of the supply chain is loyal to the CDA in the sense that each stage honestly submits its cost curves to the CDA. However, in most real world situations, the manager of each stage of a supply chain is typically selfish, rational, and intelligent and hence cares more about maximizing his/her own department's and own employees' welfare rather than welfare of the whole organization. Therefore, it should not be surprising if the managers of individual stages report untruthful cost functions to the CDA (because, in their perception, doing so may help them improve their own individual utilities/welfare). In such a situation, the behavior of the entities (namely the individual stage managers) is just like that of players in a noncooperative game. This motivates us to use an approach based on economic mechanism design [2]. The theory of economic mechanism design, in particular Groves mechanism [5, 2], basically suggests a way in which the CDA can choose a set of compensation rules that will induce the subunit managers to communicate accurate information and arrive at optimal decisions. A crucial point of these compensation rules is they do not require the organization leader to possess any additional information in order to compensate the employees or even to have knowledge of the true accuracy or completeness of information. Before applying the Groves mechanism, we set up the game model. Following is some notation that will be used subsequently:

$$
\begin{aligned}
& X_{i}=\left\{\sigma_{i}\right\}=\text { Set of values for standard deviation } \sigma_{i} \\
& X=X_{1} \times X_{2} \ldots \times X_{n} \\
& \sigma=\left(\sigma_{1}, \sigma_{2}, \ldots, \sigma_{n}\right) \\
& =\text { A standard deviation profile of stages; } \sigma \in X \\
& v_{i}: X_{i} \mapsto \Re=\text { True cost function (actual type) of stage } i \\
& w_{i}: X_{i} \mapsto \Re=\text { Reported cost function (type) of stage } i \\
& V_{i}=\left\{v_{i}\right\}=\text { Set of possible types of stage } i \\
& W_{i}=\left\{w_{i}\right\}=\text { Set of possible reported types of stage } i \\
& V=V_{1} \times V_{2} \times \ldots \times V_{n} \\
& W=W_{1} \times W_{2} \times \ldots \times W_{n} \\
& V_{-i}=V_{1} \times \ldots \times V_{i-1} \times V_{i+1} \times \ldots \times V_{n} \\
& W_{-i}=W_{1} \times \ldots \times W_{i-1} \times W_{i+1} \times \ldots \times W_{n}
\end{aligned}
$$

Let us assume that $v, w, v_{-i}$, and $w_{-i}$ represent a typical element in the sets $V, W, V_{-i}$, and $W_{-i}$, respectively. We make the following assumptions about the types of the stages.

1. The standard deviation $\sigma_{i}$ can take values from the set $\left(0, \overline{\sigma_{i}}\right]$, that is, $X_{i}=\left(0, \overline{\sigma_{i}}\right]$.

2. The true type of any stage $i$ is of the form

$$
v_{i}\left(\sigma_{i}\right)=a_{i 0}+a_{i 1} \sigma_{i}+a_{i 2} \sigma_{i}^{2} \quad \forall \sigma_{i} \in\left(0, \overline{\sigma_{i}}\right]
$$

where we assume that $v_{i}\left(\sigma_{i}\right)$ is a non-negative and non-increasing function of $\sigma_{i}$, that is, not all the three coefficients $a_{i 0}, a_{i 1}$, and $a_{i 2}$ are non-negative simultaneously.

3. For each stage $i$, it is possible to obtain an interval for each of the three coefficients $a_{i 0}, a_{i 1}$, and $a_{i 2}$. That is,

$$
a_{i 0} \in\left[\underline{a_{i 0}}, \overline{a_{i 0}}\right] ; a_{i 1} \in\left[\underline{a_{i 1}}, \overline{a_{i 1}}\right] ; a_{i 2} \in\left[\underline{a_{i 2}}, \overline{a_{i 2}}\right]
$$

These intervals are such that choosing the coefficients from the intervals will always result in a cost function $v_{i}\left(\sigma_{i}\right)$ which is non-negative and non-increasing. Also, for a given type $v_{i}\left(\sigma_{i}\right)$, the values of all the three coefficients lie in the corresponding intervals.

4. The previous assumption enables us to view the type set $V_{i}$ of stage $i$ as $\left[\underline{a_{i 0}}, \overline{a_{i 0}}\right] \times\left[a_{i 1}, \overline{a_{i 1}}\right] \times\left[\underline{a_{i 2}}, \overline{a_{i 2}}\right]$ which is a compact subset of $\Re^{3}$. The set $V$ can also 
now be viewed as a compact subset of $\Re^{3 n}$. Each point of such a compact subset of $\Re^{3 n}$ represents a unique type profile of the stages.

5. Let $\Lambda_{i}$ be a $\sigma$-algebra over the type set $V_{i}$ (a compact subset of $\Re^{3}$ ). Let $\Lambda$ be a $\sigma$-algebra over the set $V$ (a compact subset of $\Re^{3 n}$ ) which is generated by the algebras $\Lambda_{i}$.

6. We assume that $P$ is a probability measure over the $\sigma$-algebra $\Lambda$ and hence $(V, \Lambda, P)$ forms a probability space. We call $P$ as the common prior distribution over type profiles of the stages and assume that it is common knowledge among all the stages. Let $\triangle V$ represent the set of all the probability measures that can be defined over the measurable space $(V, \Lambda)$.

7. Let the manager of each stage $i$ have a belief function $p_{i}: V_{i} \mapsto \triangle V_{-i}{ }^{1}$ which describes the conjecture of stage $i$ about the types of other stages given its own type. That is, for any possible type set $\gamma_{i}$, where $\gamma_{i} \subset V_{i}$ and $\gamma_{i} \in \Lambda_{i}, p_{i}\left(. \mid \gamma_{i}\right)$ will be a probability measure over the measurable space $\left(V_{-i}, \Lambda_{-i}\right)$ and will represent what the $i^{\text {th }}$ stage manager would believe about the type sets of other stages if his own stage's type set were $\gamma_{i}$.

The Bayesian Game [10] underlying the design problem can now be described as:

$$
\begin{aligned}
\Gamma= & {\left[\left\{A_{i}\right\}_{i=1}^{n},\left\{V_{i}\right\}_{i=1}^{n},\left\{W_{i}\right\}_{i=1}^{n},\right.} \\
& \left.\left\{p_{i}(.)\right\}_{i=1}^{n},\left\{u_{i}(.)\right\}_{i=1}^{n}\right]
\end{aligned}
$$

where,

$$
\begin{aligned}
A_{i} & =\text { Manager of stage } i \text { of the supply chain } \\
V_{i} & =\text { Set of possible types of stage } i \\
& =\left[\underline{a_{i 0}}, \overline{a_{i 0}}\right] \times\left[\underline{a_{i 1}}, \overline{a_{i 1}}\right] \times\left[\underline{a_{i 2}}, \overline{a_{i 2}}\right] \\
W_{i} & =\text { Set of possible reported types of stage } i \\
& =\text { Action set for the manager of } i^{t h} \text { stage } \\
p_{i} & : V_{i} \mapsto \triangle V_{-i} \\
& =\text { Belief function for the manager of } i^{t h} \text { stage }
\end{aligned}
$$

\section{Decentralized Design using Groves Mecha- nisms}

Consider the SCOP problem shown in Figure 2. Here, the mean processing time $\mu_{i}$ for each stage $i$ is assumed to be fixed and the problem of the CDA is to determine the optimal value for standard deviation $\sigma_{i}$ for each stage $i$. Let us assume that $v_{i}\left(\sigma_{i}\right)$ is the true cost function of stage $i$,

\footnotetext{
${ }^{1} \triangle V_{-i}$ represents the set of all the probability measures that can be defined over the measurable space $\left(V_{-i}, \Lambda_{-i}\right)$
}

which is known only to the manager of stage $i$. On receiving a request from CDA, the $i^{t h}$ stage manager reports a cost function $w_{i}\left(\sigma_{i}\right)$ to the CDA which may not be the same as $v_{i}\left(\sigma_{i}\right)$.

The problem of the CDA is to find an optimal standard

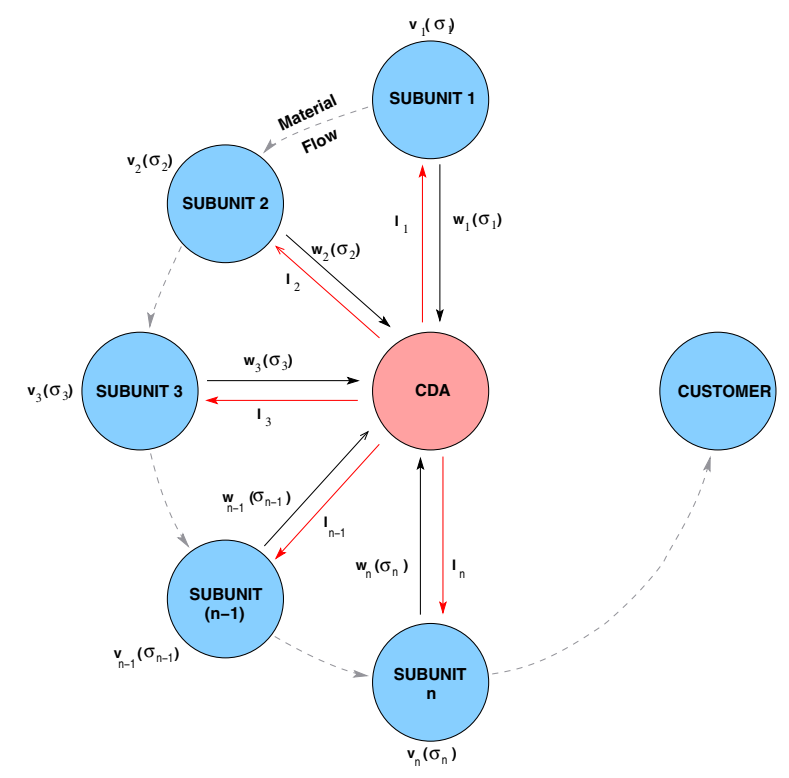

Figure 2. The decentralized design paradigm

deviation profile, say $\left(\sigma_{1}^{*}, \ldots, \sigma_{n}^{*}\right)$, of the stages that will result in the required level of delivery performance at minimum possible cost. The CDA can solve the above design problem using some optimization solver with $v_{i}()$ replaced by $w_{i}()$. Let $\left(\tilde{\sigma}_{1}^{*}, \ldots, \tilde{\sigma}_{n}^{*}\right)$ be the solution of the resulting SCOP problem. If the CDA knows in advance that the managers of the various stages are selfish, rational, and intelligent then there is no reason for the CDA to believe that that the cost functions reported are indeed their true cost functions. Therefore, blindly solving the SCOP problem will result in a non-optimal solution of the problem, that is $\left(\tilde{\sigma}_{1}^{*}, \ldots, \tilde{\sigma}_{n}^{*}\right)$ is not an optimal solution for the CDA's problem. One way to tackle this situation is for the CDA to offer an incentive $I_{i}$ to each stage $i$, which is a function of $\left(\tilde{\sigma}_{1}^{*}, \ldots, \tilde{\sigma}_{n}^{*}\right)$, i.e. $I_{i}: X_{1} \times \ldots \times X_{n} \mapsto \Re$ (see Figure 2 ), so as to induce truth revelation. In such a case, the CDA will first need to solve the problem in the usual centralized fashion by simply assuming that the reported cost functions by all the stages are indeed their true cost functions. This, in general, will give a non-optimal solution $\left(\tilde{\sigma}_{1}^{*}, \ldots, \tilde{\sigma}_{n}^{*}\right)$ of the underlying SCOP problem. Then, based upon the solution $\left(\tilde{\sigma}_{1}^{*}, \ldots, \tilde{\sigma}_{n}^{*}\right)$, the CDA will decide the incentive $I_{i}$ for each stage $i$. In such a case, the net payoff (utility) of stage $i$ will be equal to $u_{i}=I_{i}-v_{i}\left(\tilde{\sigma}_{i}^{*}\right)$ (in the literature, such a utility function is known as quasi-linear utility function [2]). Note that the payoff of stage $i$ is dependent on what 
cost functions are being reported by all the stages because the reported cost functions $w_{i}$ will decide $\left(\tilde{\sigma}_{1}^{*}, \ldots, \tilde{\sigma}_{n}^{*}\right)$ and that in turn will determine the incentive $I_{i}$ of stage $i$.

Now if the CDA can come up with an incentive structure $\left(I_{1}, \ldots, I_{n}\right)$ such that the utility $u_{i}$ of each stage $i$ gets maximized only when its reported type is the same as its true type, i.e. $w_{i}=v_{i}$, then each manager will understand this fact and has no incentive to report an untruthful cost function. The resulting solution will indeed be an optimal solution, i.e. $\left(\tilde{\sigma}_{1}^{*}, \ldots, \tilde{\sigma}_{n}^{*}\right)=\left(\sigma_{1}^{*}, \ldots, \sigma_{n}^{*}\right)$. The Groves Mechanism is a powerful way to construct such an optimal incentive structure [5].

\subsection{Groves Incentives}

The Groves incentive $I_{i}$ for stage $i$ can be computed as follows [5]:

$$
I_{i}=\alpha_{i}-\sum_{j \neq i} w_{j}\left(\tilde{\sigma}_{j}^{*}\right)
$$

where $\left(\tilde{\sigma}_{1}^{*}, \ldots, \tilde{\sigma}_{n}^{*}\right)$ is the optimal solution of the underlying SCOP problem with $v_{i}$ replaced by $w_{i} . \alpha_{i}$ is some constant which can be assumed to be the same for all the stages and can be used to normalize the value of $I_{i}$ so that it has a meaningful value. In such a case, the utility function $u_{i}$ for stage $i$ is given by

$$
\begin{aligned}
& u_{i}\left(s_{1}, \ldots, s_{n}, v_{i}\right) \\
& =\int_{v_{-i} \in V_{-i}} u_{i}\left(s_{1}, \ldots, s_{n}, v_{i}, v_{-i}\right) d p_{i}\left(v_{-i} \mid v_{i}\right) \\
& =\int_{v_{-i} \in V_{-i}} u_{i}\left(s_{1}\left(v_{1}\right), \ldots, s_{n}\left(v_{n}\right)\right) d p_{i}\left(v_{-i} \mid v_{i}\right) \\
& =\int_{v_{-i} \in V_{-i}} u_{i}\left(w_{1}, \ldots, w_{n}\right) d p_{i}\left(v_{-i} \mid v_{i}\right) \\
& =\int_{v_{-i} \in V_{-i}}\left[I_{i}\left(\tilde{\sigma}_{1}^{*}, \ldots, \tilde{\sigma}_{n}^{*}\right)-v_{i}\left(\tilde{\sigma}_{i}^{*}\right)\right] d p_{i}\left(v_{-i} \mid v_{i}\right) \\
& =\int_{v_{-i} \in V_{-i}}\left[\alpha_{i}-\sum_{j \neq i} w_{j}\left(\tilde{\sigma}_{j}^{*}\right)-v_{i}\left(\tilde{\sigma}_{i}^{*}\right)\right] d p_{i}\left(v_{-i} \mid v_{i}\right)
\end{aligned}
$$

We can prove easily that for the above utility function, the truth revealing strategy profile $s^{t}=\left(s_{i}^{t}, \ldots, s_{n}^{t}\right)$ is indeed a dominant strategy equilibrium $s^{*}=\left(s_{1}^{*}, \ldots, s_{n}^{*}\right)$ of the underlying game which means adopting the incentive structure (11) will elicit true cost functions from the stages.

\subsection{An Algorithm for Decentralized Design}

We suggest following iterative algorithm which the CDA can use to solve the SCOP problem in a decentralized manner.
Step 0: Initial Bidding Phase The CDA invites the stages to bid their cost functions and each stage bids its cost function $w_{i}\left(\sigma_{i}\right)$ which need not be its true cost function.

Step 1: Allocation Phase The CDA solves the following optimization problem. The solution of this optimization problem yields the tuple $\left(\tilde{\sigma}_{1}^{*}, \ldots, \tilde{\sigma}_{n}^{*}\right)$.

Minimize

$$
\mathcal{K}=\sum_{i=1}^{n} w_{i}\left(\sigma_{i}\right)
$$

subject to

$$
\begin{aligned}
\text { DS for end-to-end lead time } & \geq C_{p m}^{*} \\
\text { DP for end-to-end lead time } & \geq D P^{*} \\
\sigma_{i} & >0 \forall i
\end{aligned}
$$

The CDA uses the tuple $\left(\tilde{\sigma}_{1}^{*}, \ldots, \tilde{\sigma}_{n}^{*}\right)$ to compute the incentive $I_{i}$ for each stage $i$ in the following way:

$$
I_{i}=\alpha_{i}-\sum_{j \neq i} w_{j}\left(\tilde{\sigma}_{i}^{*}\right)
$$

The CDA allocates to each stage $i$ a target of attaining the $\tilde{\sigma}_{i}^{*}$ variability. The CDA also allocates a fund of $w_{i}\left(\tilde{\sigma}_{i}^{*}\right)$ to meet the expediting expenditure. The CDA also allocates an incentive $I_{i}$ to stage $i$ for its performance. Thus, the net gain of stage $i$ is $I_{i}-v_{i}\left(\tilde{\sigma}_{i}^{*}\right)$.

\section{Step 2: Iterative Bidding and Allocation}

The CDA successively invites all stages to revise and resubmit their cost functions if they wish to. Then each stage $i$ looks at its current allocation $\left(\tilde{\sigma}_{i}^{*}, I_{i}\right)$ and bids a revised cost function $w_{i}^{\prime}\left(\sigma_{i}\right)$ with the hope that in the next round its net gain will improve. Some of the stages may not revise their cost functions if they find that that their net gains may not improve.

After receiving the revised bids from each stage, the CDA again invokes Step 1 and solves the allocation problem. This initiates the next round of bidding and allocation. This iterative bidding and allocation process continues until no stage revises its cost function. The process therefore terminates when each stage bids the same cost function as in the previous round.

We assert that above algorithm will converge to a point where each stage bids its true cost function. The proof for this assertion directly follows from the fact that the truth revealing strategy is a dominant strategy for each stage under the Groves incentives structure (as already stated in Section 4.1). The convergence is guaranteed as long as the individual stages place improving bids if improvement is possible and as long as the individual stages have enough computing power to compute these improving bids. 


\section{A Case Study}

To show the efficacy of the iterative algorithm, we present a numerical experiment motivated by the outbound logistics operations in a typical automotive supply chain. Assume that a finished product (automotive vehicle) is transported from plant first by truck, then by rail, and finally by a truck to the dealer. We assume that each shipment leg is in custody of a department manager who has an idea about the delivery cost and the delivery performance of different transportation service providers available for the leg. We make the following assumptions:

1. The CDA has an ideal target of delivering a vehicle to the dealer on the $30^{t h} \pm 5$ day counting from the day it is ready for shipping at the plant.

2. There are three shipment legs in the journey of a vehicle from plant to dealer. We call these the first leg (truck), the second leg (rail road), and the third leg (truck). For each leg, there are alternate transportation service providers. We assume that there are 10 alternate service providers for each leg.

3. The mean shipment time of a vehicle on the first leg, the second leg, and the third leg of its journey is 4 days, 21 days, and 7 days respectively. For each leg, the mean is the same for all the alternate service providers available for that leg.

4. For each leg, the variability in the shipment time as well as the shipping cost for different alternate service providers is a private information of the department manager for the leg. Tables 1, 2, and 3 show the private information

5. The shipment time at each leg is normally distributed for all the service providers. Moreover, the shipment times at the three legs are mutually independent.

6. The CDA wishes to achieve $C_{p}^{*} \geq 1.8$ and $C_{p k}^{*} \geq 1.08$ for the end-to-end lead time $Y$.

7. The manager of leg $i(i=1,2,3)$, uses his/her private information to compute the true cost function as a quadratic function $v_{i}=a_{i}+b_{i} \sigma_{i}+c_{i} \sigma_{i}^{2}$, using the least square curve fitting method. For the present instance, these functions turn out to be:

$$
\begin{aligned}
& v_{1}\left(\sigma_{1}\right)=022.638-16.017 \sigma_{1}+4.015 \sigma_{1}^{2} \\
& v_{2}\left(\sigma_{2}\right)=231.085-68.624 \sigma_{2}+5.758 \sigma_{2}^{2} \\
& v_{3}\left(\sigma_{3}\right)=052.255-29.827 \sigma_{3}+5.636 \sigma_{3}^{2}
\end{aligned}
$$

\begin{tabular}{|c|c|c|}
\hline $\begin{array}{c}\text { Service } \\
\text { Provider }\end{array}$ & $\begin{array}{c}\text { Standard Deviation } \\
\left.\sigma_{1} \text { (days/unit }\right)\end{array}$ & $\begin{array}{c}\text { Shipping Cost } \\
\mathcal{K}_{1}(\$ / \text { unit })\end{array}$ \\
\hline 1 & 0.25 & 20.00 \\
2 & 0.50 & 15.00 \\
3 & 0.75 & 12.00 \\
4 & 1.00 & 10.00 \\
5 & 1.25 & 09.00 \\
6 & 1.50 & 08.00 \\
7 & 1.75 & 07.50 \\
8 & 2.00 & 07.25 \\
9 & 2.25 & 07.00 \\
10 & 2.50 & 07.00 \\
\hline
\end{tabular}

Table 1. Private information of the manager for the first leg

\begin{tabular}{|c|c|c|}
\hline $\begin{array}{c}\text { Service } \\
\text { Provider }\end{array}$ & $\begin{array}{c}\text { Standard Deviation } \\
\left.\sigma_{2} \text { (days/unit }\right)\end{array}$ & $\begin{array}{c}\text { Shipping Cost } \\
\left.\mathcal{K}_{2} \text { (\$/unit }\right)\end{array}$ \\
\hline 1 & 2.5 & 105 \\
2 & 3.0 & 70 \\
3 & 3.5 & 55 \\
4 & 4.0 & 45 \\
5 & 4.5 & 40 \\
6 & 5.0 & 35 \\
7 & 5.5 & 32 \\
8 & 6.0 & 30 \\
9 & 6.5 & 29 \\
10 & 7.0 & 28 \\
\hline
\end{tabular}

Table 2. Private information of the manager for the second leg

\begin{tabular}{|c|c|c|}
\hline $\begin{array}{c}\text { Service } \\
\text { Provider }\end{array}$ & $\begin{array}{c}\text { Standard Deviation } \\
\left.\sigma_{3} \text { (days/unit }\right)\end{array}$ & $\begin{array}{c}\text { Shipping Cost } \\
\left.\mathcal{K}_{3} \text { (\$/unit }\right)\end{array}$ \\
\hline 1 & 0.75 & 35.0 \\
2 & 1.00 & 27.0 \\
3 & 1.25 & 22.0 \\
4 & 1.50 & 19.0 \\
5 & 1.75 & 18.0 \\
6 & 2.00 & 16.0 \\
7 & 2.25 & 14.5 \\
8 & 2.50 & 13.5 \\
9 & 2.75 & 13.0 \\
10 & 3.00 & 12.5 \\
\hline
\end{tabular}

Table 3. Private information of the manager for the third leg 


\subsection{Centralized Design}

Each manager submits the true cost function and CDA just solves the single optimization problem. For the above case study, the CDA solves an appropriate optimization problem. Using the Lagrange multiplier method it is easy to show that the above optimization problem has global minimum at

$$
\sigma_{1}^{*}=0.2018 \text { days, } \sigma_{2}^{*}=0.8284 \text { days, } \sigma_{3}^{*}=0.3611 \text { days }
$$

The optimal costs for the different legs and the total optimal cost turn out be:

$$
\begin{aligned}
v_{1}\left(\sigma_{1}^{*}\right) & =19.569 \$ \text { /unit } \\
v_{2}\left(\sigma_{2}^{*}\right) & =178.190 \$ \text { /unit } \\
v_{3}\left(\sigma_{3}^{*}\right) & =042.219 \$ \text { /unit } \\
\mathcal{K}^{*} & =239.978 \text { \$/unit }
\end{aligned}
$$

\subsection{Decentralized Design}

First, let us compute the incentives and net payoffs to the managers in the case when each of them reveals the true cost function, that is $w_{i}()=.v_{i}() ; \forall i=1,2,3$. Assuming $\alpha_{1}=\alpha_{2}=\alpha_{3}=250 \$$ /unit , it is easy to see that

$$
\begin{gathered}
I_{1}^{t}=\alpha_{1}-\left(v_{2}\left(\sigma_{2}^{*}\right)+v_{3}\left(\sigma_{3}^{*}\right)\right)=29.591 \text { \$/unit } \\
I_{2}^{t}=\alpha_{2}-\left(v_{1}\left(\sigma_{1}^{*}\right)+v_{3}\left(\sigma_{3}^{*}\right)\right)=188.212 \text { \$/unit } \\
I_{3}^{t}=\alpha_{3}-\left(v_{1}\left(\sigma_{1}^{*}\right)+v_{2}\left(\sigma_{2}^{*}\right)\right)=052.241 \text { \$/unit } \\
u_{1}\left(s_{1}^{t}(.), s_{2}^{t}(.), s_{3}^{t}(.)\right)=I_{1}^{t}-v_{1}\left(\sigma_{1}^{*}\right)=10.022 \text { \$/unit } \\
u_{2}\left(s_{1}^{t}(.), s_{2}^{t}(.), s_{3}^{t}(.)\right)=I_{2}^{t}-v_{2}\left(\sigma_{2}^{*}\right)=10.022 \text { \$/unit } \\
u_{3}\left(s_{1}^{t}(.), s_{2}^{t}(.), s_{3}^{t}(.)\right)=I_{3}^{t}-v_{3}\left(\sigma_{3}^{*}\right)=10.022 \text { \$/unit }
\end{gathered}
$$

Now will show a few iterations of our algorithm.

\section{Round \# 0}

Step 0.1: Bidding Phase: In the initial bidding phase, each manager submits a cost function $w_{i}\left(\sigma_{i}\right)$ to the CDA that has higher (than true) costs. Let us assume the following initial cost functions as submitted by the managers:

$$
\begin{aligned}
& w_{1}\left(\sigma_{1}\right)=25.0-10.0 \sigma_{1}+5.0 \sigma_{1}^{2} \\
& w_{2}\left(\sigma_{2}\right)=240.0-65.0 \sigma_{2}+6.0 \sigma_{2}^{2} \\
& w_{3}\left(\sigma_{3}\right)=055.0-25.0 \sigma_{3}+6.0 \sigma_{3}^{2}
\end{aligned}
$$

It is easy to verify that $w_{i}\left(\sigma_{i}\right) \geq v_{i}\left(\sigma_{i}\right) ; \forall \sigma_{i} ; \forall i=1,2,3$. Step 0.2: Allocation Phase: In this phase, the CDA solves the following allocation problem:

Minimize

$$
\begin{aligned}
\mathcal{K} & =\sum_{i=1}^{3} w_{i}\left(\sigma_{i}\right) \\
& =320-\left(10.0 \sigma_{1}+65.0 \sigma_{2}+25.0 \sigma_{3}\right)
\end{aligned}
$$

subject to

$$
\begin{aligned}
\sigma_{1}^{2}+\sigma_{2}^{2}+\sigma_{3}^{2} & =\frac{T^{2}}{9 C_{p}^{*^{2}}}=\frac{d^{2}}{9 C_{p k}^{*^{2}}}=\frac{25}{29.16} \\
\sigma_{i} & >0 \forall i=1,2,3
\end{aligned}
$$

The solution of the above optimization problem results in

$\tilde{\sigma}_{1}^{*}=0.136$ days, $\tilde{\sigma}_{2}^{*}=0.855$ days, $\tilde{\sigma}_{3}^{*}=0.329$ days

The CDA can use the above values to compute the incentives for the managers which turn out to be:

$$
\begin{aligned}
& I_{1}=\alpha_{1}-\left(w_{2}\left(\tilde{\sigma}_{2}^{*}\right)+w_{3}\left(\tilde{\sigma}_{3}^{*}\right)\right)=13.759 \text { \$/unit } \\
& I_{2}=\alpha_{2}-\left(w_{1}\left(\tilde{\sigma}_{1}^{*}\right)+w_{3}\left(\tilde{\sigma}_{3}^{*}\right)\right)=178.832 \text { \$/unit } \\
& I_{3}=\alpha_{3}-\left(w_{1}\left(\tilde{\sigma}_{1}^{*}\right)+w_{2}\left(\tilde{\sigma}_{2}^{*}\right)\right)=37.446 \text { \$/unit }
\end{aligned}
$$

Now the manager for each leg computes his/her own payoff in following manner:

$$
\begin{aligned}
& u_{1}=I_{1}-v_{1}\left(\tilde{\sigma}_{1}^{*}\right)=-6.789 \$ / \text { unit } \\
& u_{2}=I_{2}-v_{2}\left(\tilde{\sigma}_{2}^{*}\right)=2.209 \$ / \text { unit } \\
& u_{3}=I_{3}-v_{3}\left(\tilde{\sigma}_{3}^{*}\right)=-0.598 \text { \$/unit }
\end{aligned}
$$

\section{Round \# 1}

Step 1.1: Bidding Phase: Observe that in the previous round, the net payoff of each manager is less than what he/she would have got with a truth revealing strategy. However, it is not possible for a manager to compute his/her payoff under a truth revealing strategy profile a priori because he/she does not know the actual cost functions of the other managers. Therefore, each manager just tries to maximize his/her own payoff by hiking the costs. After knowing the incentives $I_{i}$ and variability target $\tilde{\sigma}_{i}^{*}$ from the CDA in the previous round, each manager will further revise his/her cost function in a way that can hopefully fetch him/her more payoff. Let us assume that the managers bid the following cost functions in this round:

$$
\begin{aligned}
& w_{1}^{\prime}\left(\sigma_{1}\right)=030.0-08.0 \sigma_{1}+7.0 \sigma_{1}^{2} \\
& w_{2}^{\prime}\left(\sigma_{2}\right)=250.0-60.0 \sigma_{2}+7.0 \sigma_{2}^{2} \\
& w_{3}^{\prime}\left(\sigma_{3}\right)=060.0-20.0 \sigma_{3}+7.0 \sigma_{3}^{2}
\end{aligned}
$$

It is easy to verify that $w_{i}^{\prime}\left(\sigma_{i}\right) \geq w_{i}\left(\sigma_{i}\right) ; \forall \sigma_{i} ; \forall i=1,2,3$. Step 1.2: Allocation Phase: In this phase, the CDA solves an appropriate allocation problem and the solution of the problem results in:

$\tilde{\sigma}_{1}^{*}=0.127$ days, $\tilde{\sigma}_{2}^{*}=0.954$ days, $\tilde{\sigma}_{3}^{*}=0.318$ days

Incentive and payoff for the manager of each leg turns out to be the following.

$$
I_{1}^{\prime}=\alpha_{1}-\left(w_{2}^{\prime}\left(\tilde{\sigma}_{2}^{*}\right)+w_{3}^{\prime}\left(\tilde{\sigma}_{3}^{*}\right)\right)=-3.444 \$ \text { /unit }
$$




$$
\begin{aligned}
& I_{2}^{\prime}=\alpha_{2}-\left(w_{1}^{\prime}\left(\tilde{\sigma}_{1}^{*}\right)+w_{3}^{\prime}\left(\tilde{\sigma}_{3}^{*}\right)\right)=166.560 \text { \$/unit } \\
& I_{3}^{\prime}=\alpha_{3}-\left(w_{1}^{\prime}\left(\tilde{\sigma}_{1}^{*}\right)+w_{2}^{\prime}\left(\tilde{\sigma}_{2}^{*}\right)\right)=21.804 \text { \$unit } \\
& u_{1}^{\prime}=I_{1}^{\prime}-v_{1}\left(\tilde{\sigma}_{1}^{*}\right)=-24.109 \text { \$/unit } \\
& u_{2}^{\prime}=I_{2}^{\prime}-v_{2}\left(\tilde{\sigma}_{2}^{*}\right)=-4.259 \$ \text { unit } \\
& u_{3}^{\prime}=I_{3}^{\prime}-v_{3}\left(\tilde{\sigma}_{3}^{*}\right)=-21.529 \text { \$/unit }
\end{aligned}
$$

\section{Round \# 2}

Step 2.1: Bidding Phase: Having realized the fact that hiking the cost is not improving payoffs, each manager slashes the cost in this round. Let the bids received by the CDA in this round be as follows:

$$
\begin{aligned}
& w_{1}^{\prime \prime}\left(\sigma_{1}\right)=023.0-14.0 \sigma_{1}+4.5 \sigma_{1}^{2} \\
& w_{2}^{\prime \prime}\left(\sigma_{2}\right)=235.0-67.0 \sigma_{2}+6.0 \sigma_{2}^{2} \\
& w_{3}^{\prime \prime}\left(\sigma_{3}\right)=054.0-27.0 \sigma_{3}+6.0 \sigma_{3}^{2}
\end{aligned}
$$

It is easy to verify that $w_{i}\left(\sigma_{i}\right) \geq w_{i}^{\prime \prime}\left(\sigma_{i}\right) \geq$ $v_{i}\left(\sigma_{i}\right) ; \forall \sigma_{i} ; \forall i=1,2,3$

Step 2.2: Allocation Phase: Solving the CDA's problem in a way similar to the previous two rounds, we get the following values for the various quantities.

$$
\begin{aligned}
\tilde{\sigma}_{1}^{*} & =0.1828 \text { days, } \tilde{\sigma}_{2}^{*}=0.8419 \text { days, } \tilde{\sigma}_{3}^{*}=0.3392 \text { days } \\
I_{1}^{\prime \prime} & =\alpha_{1}-\left(w_{2}^{\prime \prime}\left(\tilde{\sigma}_{2}^{*}\right)+w_{3}^{\prime \prime}\left(\tilde{\sigma}_{3}^{*}\right)\right)=21.624 \text { \$/unit } \\
I_{2}^{\prime \prime} & =\alpha_{2}-\left(w_{1}^{\prime \prime}\left(\tilde{\sigma}_{1}^{*}\right)+w_{3}^{\prime \prime}\left(\tilde{\sigma}_{3}^{*}\right)\right)=183.878 \text { \$/unit } \\
I_{3}^{\prime \prime} & =\alpha_{3}-\left(w_{1}^{\prime \prime}\left(\tilde{\sigma}_{1}^{*}\right)+w_{2}^{\prime \prime}\left(\tilde{\sigma}_{2}^{*}\right)\right)=46.564 \text { \$unit } \\
u_{1}^{\prime \prime} & =I_{1}^{\prime \prime}-v_{1}\left(\tilde{\sigma}_{1}^{*}\right)=1.780 \$ \text { /unit } \\
u_{2}^{\prime \prime} & =I_{2}^{\prime \prime}-v_{2}\left(\tilde{\sigma}_{2}^{*}\right)=6.487 \text { \$unit } \\
u_{3}^{\prime \prime} & =I_{3}^{\prime \prime}-v_{3}\left(\tilde{\sigma}_{3}^{*}\right)=3.779 \text { \$/unit }
\end{aligned}
$$

Thus, we see that as the cost function reported by a manager approaches the true cost function, the payoff for the manager improves and and attains maximum value when the manager reports the true cost function.

\section{Conclusions}

In this paper, we have use of game theory and mechanism design theory in proposing a new, realistic, and promising way of designing supply chains. We showed that a supply chain network is best viewed as a conglomeration of semi-autonomous or near-autonomous entities, where the individual entities have their own individual goals and utilities to optimize which may not necessarily result in optimizing a system-wide objective. This leads to a noncooperative game model which we called the supply chain design game. We then showed that mechanism design theory, in particular, Groves mechanisms, provides a natural framework for modeling and analyzing the supply chain design game. The application of Groves mechanism design approach to supply chains enables a central design authority to determine incentives/penalties which induce truth revelation by individual entities of the supply chain. This in turn leads to the design of high performance supply chains at minimum cost. We showed the application of the proposed approach to the design of a stylized version of a typical three stage automotive distribution process.

The research has opened up a new approach for supply chain design which is more realistic and natural. The approach needs to be developed into a comprehensive design methodology for supply chain networks and this calls for addressing many questions. (1) With modern supply chains becoming information driven and with technologies such as RFID tags driving revolutionary possibilities, how can one leverage decentralized approaches such as proposed in the paper towards a better design/operation of supply chains? (2) What are the limits of anarchical behavior of selfish agents in the supply chain design game? How can game theory and mechanism design be applied to allow maximum freedom to individual entities and yet maximize overall, social benefits?

\section{Acknowledgments}

The first two authors would like to acknowledge the generous support of General Motors R \& D Center, Warren, Michigan, USA, for supporting this research.

\section{References}

[1] G. Cachon and S. Netesssine. Game theory in supply chain analysis. In D. Simchi-Levi, D. Wu, and Shen, editors, Supply Chain Analysis in the eBusiness Area. Kluwer Academic Publishers, 2005.

[2] A. M. Colell, M. D. Whinston, and J. R. Green. Microeconomic Theory. Oxford University Press, New York, 1995.

[3] M. Fan, J. Stallaert, and A. Whinston. Decentralized mechanism design for supply chain organizations using an auction market. Information Systems Research, 14(1):1-22, 2003.

[4] D. Garg, Y. Narahari, and N. Viswanadham. Design of six sigma supply chains. IEEE Transactions on Automation Science and Engineering, 1(1):38-57, July 2004.

[5] T. Groves. Incentives in teams. Econometrica, 41:617-631, 1973.

[6] V. E. Kane. Process capability indices. Journal of Quality Technology, 18:41-52, 1986.

[7] S. Karabuk and D. Wu. Incentive schemes for semiconductor capacity allocation: A game theoretic analysis. Technical report, Department of Industrial and Systems Engineering, Lehigh University, http://www.lehigh.edu/sdw1/, 2004.

[8] S. Kotz and C. R. Lovelace. Process Capability Indices in Theory and Practice. Arnold, 1998.

[9] E. Kutanoglu and D. Wu. Collaborative resource planning with distributed agents. Technical report, Department of Industrial and Systems Engineering, Lehigh University, http://www.lehigh.edu/sdw1/, 2004

[10] R. B. Myerson. Game Theory: Analysis of Conflict. Harvard University Press, Cambridge, Massachusetts, 1997. 